\title{
"EL PUDOR ME OBLIGA A CALLAR". DE LA BIENSÉANCE DANS LA TRADUCTION D'UNE HISTOIRE TRAGIQUE DE BELLEFOREST PAR AGUSTÍN PÉREZ ZARAGOZA DANS LA GALERÍA FÚNEBRE (1831)
}

\author{
Justine Pédeflous \\ Université Paris IV-Sorbonne
}

\begin{abstract}
RÉSUMÉ: Cet article entend mettre en valeur un aspect du travail de traduction effectué par Agustín Pérez Zaragoza dans le recueil gothique intitulé Galería fúnebre (1831), longtemps considéré comme une æuvre originale de l'auteur. Dans cet ouvrage, Pérez Zaragoza s'inspire non seulement d'un recueil gothique français, les Ombres sanglantes (1820), de Pierre Cuisin mais également des Histoires tragiques (1566), de François de Belleforest. Or, sa compréhension de la traduction est plus proche du concept d'adaptation, notamment dans sa prise en compte de la sensibilité féminine et bourgeoise de son lectorat. L'exemple de la traduction de l'histoire XXIII de Belleforest nous permettra de montrer dans quelle mesure la pudibonderie du traducteur ou son souci de la bienséance transforme le texte-source en une véritable réécriture.
\end{abstract}

MOTS CLÉS: Pérez Zaragoza (Agustín), littérature gothique, Belleforest (François de), Bandello (Matteo), histoires tragiques, érotisme.

\section{"EL PUDOR ME OBLIGA A CALLAR". DE LA DECENCIA EN LA TRADUCCIÓN DE UNA HISTORIA TRÁGICA DE BELLEFOREST POR AGUSTÍN PÉREZ ZARAGOZA EN LA GALERÍA FÚNEBRE (1831)}

\footnotetext{
RESUMEN: Este artículo pretende poner de relieve una dimensión del trabajo de traducción efectuado por Agustín Pérez Zaragoza en la colección gótica titulada Galería fúnebre (1831), que fue considerada durante mucho tiempo como una obra original del autor. En esta obra, Pérez Zaragoza se inspira no sólo en una colección gótica francesa, las Ombres sanglantes (1820), de Pierre Cuisin sino también en las Histoires tragiques (1566), de François de Belleforest. Ahora bien, su comprensión de
} 
la traducción se parece más bien al concepto de adaptación, en particular en su consideración de la sensibilidad femenina y burguesa de su lectorado. El ejemplo de la traducción de la historia XXIII de Belleforest nos permitirá mostrar en qué medida la pudibundez del traductor o su atención a la decencia transforma el texto-fuente en una verdadera reescritura.

PALABRAS CLAVE: Pérez Zaragoza (Agustín), literatura gótica, Belleforest (François de), Bandello (Matteo), historias trágicas, erotismo.

La Galería fúnebre, recueil de vingt-quatre nouvelles ${ }^{1}$ en douze tomes, paru en 1831 sous la plume d'Agustín Pérez Zaragoza, est considérée comme l'ouvrage gothique de référence en Espagne, que cela soit en bonne ou en mauvaise part. Ainsi, Larra, dans son célèbre article "¿Quién es el público y dónde se encuentra?", prend comme exemple typique du mauvais goût espagnol de l'époque, les lecteurs de la Galería fúnebre ${ }^{2}$; plus tard, Mesonero Romanos fera de Pérez Zaragoza le modèle de l'écrivain médiocre de la période ${ }^{3}$. Malgré ce sévère jugement sur la qualité de l'œuvre, selon plusieurs témoignages, le recueil de Pérez Zaragoza a été un véritable best-seller à son époque ${ }^{4}$.

Or, le paradoxe constitue un élément clé de la réception de ce texte. Il faudra ainsi attendre 1977, avec l'édition critique de Luis Alberto de Cuenca, pour qu'il sorte de l'oubli. Cependant, jusqu'en 2007, il sera majoritairement considéré comme une œuvre originale, dont certains récits s'inspiraient des Novelle de Matteo Bandello. Ce n'est qu'en 2007 que María José Alonso Seoane montre qu'il s'agit en partie de la traduction d'une œuvre française de 1821, les $\mathrm{Om}$ bres sanglantes, de Pierre Cuisin, et en partie de la traduction des Histoires tragiques, de François de Belleforest, qui constituent une traduction libre des nouvelles de Bandello (Alonso Seoane 2007). Elle a également découvert qu'une autre traduction de l'œuvre de Pierre Cuisin avait été publiée en Espagne un an avant celle de Pérez Zaragoza, mais qu'elle n'a pas eu le même succès que celle-ci et est complètement tombée dans l'oubli (Castellanos y Anento 1830).

1. Pérez Zaragoza intitule vingt-et-un de ses récits "historia trágica", et trois "novela".

2. “Será el público el que compra la Galería fúnebre de espectros y sombras ensangrentadas y las poesías de Salas [...]?”, en “¿Quién es el público y dónde se encuentra?”, El Pobrecito Hablador, 17 août 1832 (Larra 1995: 36).

3. Ramón Mesonero Romanos, Memorias de un setentón. Madrid: Tebas. 1975 (1881): 268269 (Cuenca 1977: 16).

4. "Volvemos a asegurar que con esta publicación le ha caído al señor Zaragoza la lotería", Cartas españolas, I, juin 1831, p. 228 (Cuenca 1977: 18). 
Une des différences entre les deux textes pouvant contribuer à expliquer leur postérité inégale est leur rapport à la traduction: alors que les deux premiers traducteurs revendiquaient leur statut de traducteur, nulle part dans la Galería fúnebre, on ne trouve de référence à l'un ou l'autre des textes-sources de Pérez Zaragoza, qui se déclare "autor" de l'œuvre: il précède ainsi son œuvre d'un "Prolegómeno del autor a sus lectores".

Il est certain que Pérez Zaragoza inclut dans son ouvrage plusieurs nouvelles de son $\mathrm{cru}^{5}$ mais il est intéressant de voir dans les textes traduits eux-mêmes un travail d'adaptation et de recomposition qui transforme souvent de manière significative le texte-source. Les modifications les plus symptomatiques concernent la dimension sensuelle et sexuelle des textes-sources. On constate en effet chez le traducteur une propension à la "dés-érotisation", dont il conviendra d'étudier les effets sur la signification globale du texte. Nous nous demanderons ainsi si cette dés-érotisation répond simplement à une exigence d'adaptation du texte au contexte de sa traduction (censure institutionnelle, goût du public) ou bien si elle est au centre d'un véritable acte de réécriture de la part du traducteur.

\section{La Galería fúnebre et les histoires tragiques}

Les nouvelles de Bandello ont ouvert la voie en France à un nouveau genre littéraire qui se développe entre 1559, date de parution de la première traduction de Bandello par Pierre Boaistuau et les années 1650, où paraissent les dernières histoires tragiques signées de l'évêque Jean-Pierre Camus. Thierry Pech définit ainsi les caractéristiques du genre: 1'histoire tragique se développe sur un mode grave, à l'inverse de la tradition du récit bref dit "réaliste", à savoir le fabliau ou le joyeux devis (Pech 2000: 42). Un des critères d'identification de ces récits est la recherche de pathos qui influencera l'esthétique paroxystique du théâtre baroque. Par ailleurs, le personnel narratif est tiré majoritairement de la noblesse. Le plus souvent, la narration possède une structure ternaire comportant une introduction générale, un récit et une conclusion qui tire les leçons de l'intrigue. Cette composition correspond à ce que Thierry Pech nomme le "récit de loi" qui se divise en trois parties: expression de la loi, récit de la transgres-

5. "Novela: Los castillos en el aire" (vol. VIII); "Historia trágica 17: Los dos crímenes" (vol. VIII); "Historia trágica 18: Varinka, o efectos de una mala educación. Historia rusa" (vol. IX); "Historia trágica 21: El judio bienhechor, o Elisa y Teodoro" (vol. X, XI, XII). 
sion, représentation de la punition (2000: 209). Cette structure se prête naturellement à l'édification qui est toujours revendiquée dans les prologues mais que les auteurs mettent en œuvre de diverses façons. Enfin, la thématique des histoires tragiques est plus réduite que celle des recueils traditionnels de nouvelles dans la mesure où elles mettent en scène des amours malheureuses, voire destructrices: en cela, les histoires tragiques s'opposent aux traditions littéraires du pétrarquisme et du néo-platonisme.

Concernant l'évolution du genre au fur et à mesure des auteurs, Thierry Pech indique que si Boaistuau avait eu tendance à alléger la représentation de la violence, à estomper le caractère scandaleux de certaines scènes, à ajouter des discours, des lettres et des analyses psychologiques, Belleforest ajoutent quant à lui des plaintes, des exemples historiques et surtout des considérations morales. Avec les histoires tragiques, renaît le vieux débat sur le danger d'utiliser la littérature à des fins édifiantes, à cause de la création d'un système de sympathie qui pourrait court-circuiter la démonstration rationnelle de la loi. S'inspirant du récit criminel avec lequel elle partage la structure tripartite, l'histoire tragique se distingue néanmoins de lui par l'intérêt qu'elle porte aux circonstances du crime et aux motifs des criminels, ce qui peut créer chez le lecteur de la compassion au lieu de faire naître de la terreur.

Si l'expression de la loi est au-dessus de toute ambigüité -le discours du narrateur dans le prologue d'une histoire tragique est toujours caractérisé par un ton sentencieux-, en revanche sa mise en œuvre dans le récit peut révéler des failles dans le processus exemplaire. Parfois la faute mise en avant dans la narration n'est pas la même que celle annoncée dans l'introduction ${ }^{6}$; d'autres fois le retour à l'ordre n'est pas entièrement satisfaisant, car certains crimes restent impunis; enfin, certains récits assument leur polyphonie en mettant en avant plusieurs options judiciaires, ce qui a pour effet de brouiller les repères axiologiques ${ }^{7}$. Enfin, Jean-Claude Arnould signale l'écart qu'il pouvait y avoir entre le projet édifiant de l'auteur et la réception d'un public plus friand des scènes licencieuses ou violentes que du récit-cadre moralisant (Arnould 2003: 205).

6. Ainsi dans l'histoire IV de Boaistuau, si l'introduction annonçait l'horreur de l'adultère, la fin du récit met plutôt l'accent sur l'horreur de la vengeance du mari (Pech 2000: 209).

7. Dans l'histoire XI de Rosset (Histoires mémorables et tragiques de notre temps, 1623), les uns et les autres expriment leur opinion sur le crime commis (Pech 2000: 255). 
Agustín Pérez Zaragoza tire douze de ses nouvelles du tome second des Histoires tragiques publiées par Belleforest en 1566: nous reproduisons sur le tableau ci-dessous les correspondances entre les nouvelles de Bandello, les histoires tragiques de Belleforest et celles de Pérez Zaragoza. Concernant la question de la connaissance de Pérez Zaragoza des histoires tragiques, il faut faire l'hypothèse qu'il a eu accès à la traduction de Belleforest lors de son séjour en France. Ainsi, contrairement à la France, la réception des nouvelles de Bandello en Espagne est plus réduite: on ne trouve qu'une traduction, datant de 1589 et rééditée en 1603 (Zerari Penin 2012: 278) ${ }^{8}$. Cette traduction comprend quatorze nouvelles, dont aucune ne figurera chez Pérez Zaragoza, ce qui confirme la médiation française. La connaissance de Bandello dans l'Espagne du début du XIXème siècle devait être très réduite dans la mesure où l'on ne trouve dans aucune des annonces ou critiques contemporaines de la Galería fúnebre l'indice de sa source italienne.

8. Je remercie chaleureusement Maria Zerari Penin de m'avoir fourni cet article et pour ses précieux conseils. 


\begin{tabular}{|c|c|c|}
\hline Novelle, de Matteo Bandello (1554) & $\begin{array}{l}\text { Histoires tragiques, de François de Belle- } \\
\text { forest, tome second (1566) }\end{array}$ & $\begin{array}{l}\text { Galería fúnebre, de Pérez Za- } \\
\text { ragoza (1831) }\end{array}$ \\
\hline $\begin{array}{l}\text { Prima parte, Novelle LV: Un caste- } \\
\text { llano trovata la moglie in adulterio } \\
\text { col suo signore gli ammazza, ond'e- } \\
\text { gli con molti altri è miserabilmente } \\
\text { morto }\end{array}$ & $\begin{array}{l}\text { Histoire XXIII (tome II): Des grandes } \\
\text { cruautés advenues pour l'adultère d'un des } \\
\text { seigneurs de Nocere avec la femme du châ- } \\
\text { telain au fort de ladite cité }\end{array}$ & $\begin{array}{l}\text { Vol. II: Historia trágica 4: El al- } \\
\text { calde de Nóchera, o Nicolo, } \\
\text { señor de Forliño }\end{array}$ \\
\hline $\begin{array}{l}\text { Prima parte, Novelle XXVI: Il signor } \\
\text { Antonio Bologna sposa la duchessa di } \\
\text { Malfi e tutti dui sono ammazzati }\end{array}$ & $\begin{array}{l}\text { Histoire XIX: L'infortuné mariage du seig- } \\
\text { neur Antonio Bologne, avec la Duchesse de } \\
\text { Malfi, et la mort piteuse de tous les deux }\end{array}$ & $\begin{array}{l}\text { Vol. III: Historia trágica 6: La } \\
\text { duquesa de Malfi }\end{array}$ \\
\hline $\begin{array}{l}\text { Prima parte, Novella XXXIII: Dui } \\
\text { amanti si trovano la notte insieme, e il } \\
\text { giovine di gioia si muore e la fanciu- } \\
\text { lla di dolor s'accora }\end{array}$ & $\begin{array}{l}\text { Histoire XXII: De deux amants, lesquels se } \\
\text { trouvant la nuit ensemble, l'un meurt de } \\
\text { joie, et l'autre le suit suffoqué de trop de } \\
\text { douleur }\end{array}$ & $\begin{array}{l}\text { Vol. IV: Historia trágica } 8: \text { Ca- } \\
\text { mila y Livio, o los efectos de un } \\
\text { amor desgraciado }\end{array}$ \\
\hline $\begin{array}{l}\text { Prima parte, Novella LVII: Una cor- } \\
\text { tesia usata da Mansor re e pontefice } \\
\text { maomettano di Marocco ad un povero } \\
\text { pescatore suo soggetto }\end{array}$ & $\begin{array}{l}\text { Histoire XXIV: De quelle courtoisie usa le } \\
\text { Roi de Marroco (sic) envers un pauvre pé- } \\
\text { cheur, sien sujet, qui l'avait logé, le Roi s'é- } \\
\text { tant égaré à la chasse }\end{array}$ & $\begin{array}{l}\text { Vol. IV: Novela: El pescador, o } \\
\text { rasgo de nobleza de Mansor, rey } \\
\text { de Marruecos }\end{array}$ \\
\hline $\begin{array}{l}\text { Prima parte, Novella XXVIII: Varii } \\
\text { accidenti e pericoli grandissimi avve- } \\
\text { nuti a Cornelio per amor d'una gio- } \\
\text { vane }\end{array}$ & $\begin{array}{l}\text { XXVI: Divers accidents advenus à } \\
\text { llhomme Milanais pour l'amour } \\
\text { nne favorite }\end{array}$ & $\begin{array}{l}\text { Vol. V: Historia trágica 11: Cor- } \\
\text { nelio y Camila, o locuras de } \\
\text { amor }\end{array}$ \\
\hline $\begin{array}{l}\text { Prima parte, Novella V: Quanto scal- } \\
\text { tritamente Bindoccia beffa il suo ma- } \\
\text { rito che era fatto geloso }\end{array}$ & $\begin{array}{l}\text { Histoire XX: Vie désordonnée de la Com- } \\
\text { tesse de Célant, et comme ayant fait meur- } \\
\text { trir el Comte de Mussine, elle fut décapitée } \\
\text { à Milan }\end{array}$ & $\begin{array}{l}\text { Vol. VI: Historia trágica 13: } \\
\text { Blanca María, o la condesa de } \\
\text { Celán }\end{array}$ \\
\hline $\begin{array}{l}\text { Prima parte, Novella XLIX: Anselmo } \\
\text { Salimbene magni-ficamente operando } \\
\text { libera il suo nemico da la morte e la } \\
\text { sorella di quello prende per moglie }\end{array}$ & $\begin{array}{l}\text { Histoire XXI: Acte généreux d'un Gentil- } \\
\text { homme, lequel délivra son ennemi de la } \\
\text { mort, et l'autre qui lui fit présent de la sœur, } \\
\text { de laquelle il le savait être amoureux }\end{array}$ & $\begin{array}{l}\text { Vol. VI: } N \\
\text { Salimbene }\end{array}$ \\
\hline $\begin{array}{l}\text { Prima parte, Novella VIII: Giulia da } \\
\text { Gazuolo, essendo per forza violata, in } \\
\text { Oglio si getta, ove mori }\end{array}$ & $\begin{array}{l}\text { Histoire XXV: Mort piteuse de Julie de Ga- } \\
\text { zole, laquelle se noya de dépit pour se voir } \\
\text { violée }\end{array}$ & $\begin{array}{l}\text { Vol. VII: Historia trágica 14: La } \\
\text { bella mantuana o Julia de Ga- } \\
\text { zola }\end{array}$ \\
\hline $\begin{array}{l}\text { Seconda parte, Novella V: Fabio ro- } \\
\text { mano è da Emilia per gelosia am- } \\
\text { mazzato a ciò che un'altra per moglie } \\
\text { non pigliasse, ed ella sovra di lui su- } \\
\text { bito s'uccide }\end{array}$ & $\begin{array}{l}\text { Histoire XXX: Emilie Damoiselle Ro- } \\
\text { maine, tua Fabio son ami, afin qu'il n'en } \\
\text { épousât une autre qu'on lui avait donnée, } \\
\text { puis elle-même s'occit sur lui }\end{array}$ & $\begin{array}{l}\text { Vol. VII: Historia trágica } 15 \text { : } \\
\text { Emilia y Fabio, o tristes efectos } \\
\text { del amor }\end{array}$ \\
\hline $\begin{array}{l}\text { Prima parte, Novella XIV: Antonio } \\
\text { Perillo dopo molti travagli sposa la } \\
\text { sua amante, e la prima notte sono dal } \\
\text { folgore morti }\end{array}$ & $\begin{array}{l}\text { Histoire XXVII: D'un jeune homme Napo- } \\
\text { litain, lequel la nuit de ses noces couché } \\
\text { avec sa femme, fut foudroyé, après avoir } \\
\text { souffert beaucoup pour celle qu'il épousa }\end{array}$ & $\begin{array}{l}\text { Vol. VII: Historic } \\
\text { Carmosina y Max }\end{array}$ \\
\hline $\begin{array}{l}\text { Terza parte, Novella XXI: Uno } \\
\text { schiavo battuto dal padrone ammazza } \\
\text { la padrona con i figliuoli e poi se } \\
\text { stesso precipita da un'alta torre }\end{array}$ & $\begin{array}{l}\text { Histoire XXXI: Un esclave More étant } \\
\text { battu de son maître, s'en vengea avec une } \\
\text { cruauté grande, et fort étrange }\end{array}$ & $\begin{array}{l}\text { Vol. IX: Historia trágica } 19: \text { El } \\
\text { esclavo moro, o crueldad sobre } \\
\text { crueldad }\end{array}$ \\
\hline $\begin{array}{l}\text { Terza parte, Novella XXIX: Sotto spe- } \\
\text { cie di far alcuni incantesimi, uno sco- } \\
\text { lare di paura se ne more, essendo in } \\
\text { una sepoltura }\end{array}$ & $\begin{array}{l}\text { Histoire XXXII: Un écolier à Bologne pen- } \\
\text { sant faire quelque enchantement, mourut de } \\
\text { peur, étant dans un tombeau au Cimetière }\end{array}$ & $\begin{array}{l}\text { Vol. IX: Historia trágica 20: } \\
\text { Clotilde y Lirinio }\end{array}$ \\
\hline
\end{tabular}




\section{La dés-érotisation du texte comme caractéristique du travail de traduction dans la Galería fúnebre}

A l'inverse de la traduction de 1589 , qui était une traduction fidèle, certainement due en partie à la proximité entre la date de parution de la source et celle de la traduction, Pérez Zaragoza propose une traduction des Histoires tragiques presque trois siècles après leur écriture, ce qui entraîne de nombreuses modifications du texte. Pérez Zaragoza choisit de ne pas traduire toutes les nouvelles du recueil et en modifie les titres afin de les adapter au goût du jour. Si Belleforest avait globalement conservé la forme des titres de Bandello, à savoir un titre long, résumant l'intrigue, annonçant même parfois la fin du récit, et ne nommant pas nécessairement les protagonistes ("Un écolier à Bologne pensant faire quelque enchantement, mourut de peur, étant dans un tombeau au Cimetière", Histoire XXXII: 343-3649), Pérez Zaragoza choisit de mettre les personnages en avant en laissant de côté l'intrigue: le titre de l'histoire tragique XXXII devient ainsi "Clotilde y Lirinio" (Historia trágica 20, vol. IX: 157-223). L'accent mis sur les personnages au détriment de l'intrigue, correspond à l'évolution des codes d'écriture et de réception entre l'original du XVIème siècle et la traduction du XIXème siècle. En effet, si au XVIème siècle, le personnage était proche du type servant de support au développement d'une intrigue à visée moralisante, à partir de la fin du XVIIIème siècle, l'accent mis par les Lumières sur l'individu et les nouvelles théories épistémologiques fondées sur l'expérience des sens entraîne en littérature, et plus particulièrement dans le roman et la nouvelle, une focalisation sur le personnage : l'intrigue passe ainsi au second plan au profit de l'analyse psychologique de l'intériorité des personnages. Parallèlement, le public, comprenant de plus en plus de femmes, et plus prompt à l'identification avec les personnages littéraires, devient demandeur de sentimentalité. Ainsi, les changements de titres de Pérez Zaragoza correspondraient à une volonté d'adaptation d'une matière ancienne au goût du jour.

De plus, il est fréquent que l'auteur ajoute dans le titre un personnage féminin qui ne figurait pas dans le titre original ou qui y figurait au second plan: par exemple Pérez Zaragoza remplace "Acte généreux d'un Gentilhomme, lequel délivra son ennemi de la mort, et l'autre qui lui fit présent de la sœur; de laquelle il le savait être amoureux" par "Angélica, o los Salimbenes y Montanes". Ici le personnage féminin, qui n'avait qu'un rôle passif dans le titre original, passe au premier plan

9. Le titre d'origine était "Sotto specie di far alcuni incantesimi, uno scolare di paura se ne more, essendo in una sepoltura" (Novella XXIX, Terza parte). 
jusqu'à remplacer le "généreux Gentilhomme", ce qui conditionne l'interprétation du récit. On peut y voir une stratégie d'écriture visant à intéresser le public féminin, auquel il s'adresse de façon explicite dans le paratexte: l'auteur traduit en effet dans son "Introducción analítica" une scène incluse par Cuisin dans son prologue, mettant en scène une jeune fille passionnée par les œuvres terrifiantes en train de lire son propre livre ${ }^{10}$.

Outre la modification des titres, les transformations les plus fréquentes et significatives concernent les connotations érotiques et sexuelles, que Pérez Zaragoza tend à estomper voire éliminer de sa traduction. On observe cette tendance dans la traduction des récits de Cuisin. Par exemple, dans "La morada de un parricida" 11 , le traducteur supprime une scène entière entre le héros et sa maîtresse ${ }^{12}$ pour ne garder que l'allusion à des gages d'amour indiquant leur engagement mutuel mais purement platoniques: "retratos, cabellos, anillos, cifras amorosas, de todo se habia valido su amor" (Pérez Zaragoza 1977 [1831]: 96) ${ }^{13}$. Le traducteur supprimera également l'allusion à des "songes délicieux" (Cuisin 1820: 39) pouvant renvoyer à une réalité érotique. Allant dans le sens de cette dés-érotisation, Pérez Zaragoza remplace la référence trop sensuelle au "lit nuptial" (Cuisin 1820: 42) par une expression plus neutre: "enlace nupcial" (98). Ce phénomène de neutralisation de l'érotisme semble être une constante de l'écriture de Pérez Zaragoza car on le retrouve dans "Miladi Herwort", à propos de la jeune Clarisa, qui traverse avec sa mère un bois réputé dangereux la nuit, le segment "le sein douloureusement oppressé" (Cuisin 1820: 234), se transforme en "oprimido dolorosamente su corazón" (82). Le traducteur supprime donc les éléments érotiques ou les neutralise au moyen de termes renvoyant à des réalités plus abstraites ou générales et moins corporelles.

10. Cette scène se trouve aux pages 22 à 24 de l'introduction de Cuisin aux Ombres sanglantes, et sa traduction aux pages 62 et 63 de l'édition de 1977 de Cuenca dont nous indiquerons les pages entre parenthèses.

11. "Historia trágica 2: La morada de un parricida, o triunfo del remordimiento " (vol. I) est la traduction de "Premières Ombres. La demeure d'un parricide ou le triomphe du remords", Ombres sanglantes, tome I.

12. "Instants délicieux, prémices d'amour où deux jeunes cœurs s'abandonnent à la virginité, à la pureté de leurs sentiments ! vous êtes bien pour l'homme le suprême bonheur sur la terre. En effet, qui ne se rappelle avec transport, avec attendrissement le premier baiser virginal de sa jeunesse ! le moment divin où une bouche adorée vous balbutia, les yeux baissés: oui, je vous aime... où plus heureux encore, on serra dans ses bras, on pressa sur son sein le sein ému de sa maîtresse, et enfin où l'on échangea, dans l'excès de son délire, les gages ordinaires de l'amour !!... Ces moments inappréciables avaient été connus, avaient été sentis par Amédée, par Blanche, sa fidèle et tendre amante." (Cuisin 1820 : 37-38).

13. Par la suite, toutes les citations en espagnol sont de Pérez Zaragoza. 
Concernant la traduction des Histoires tragiques, c'est dans "El alcalde de Nóchera"14, récit dans lequel l'adultère est au centre de l'intrigue, que le travail de dés-érotisation du traducteur est le plus visible et a des conséquences sur l'ensemble du texte. En effet, dès le début de la nouvelle, le traducteur se montre infidèle au texte-source en transformant la "corne d'abondance de cocuage" (Belleforest 1566: 160) $)^{15}$ en "corneta de Amaltea" (149) (corne d'Amalthée ${ }^{16}$ ). Ici, afin de rendre son texte acceptable d'un point de vue moral, Pérez Zaragoza trahit sa source en ne gardant qu'un seul élément, celui de la corne d'abondance, et en éliminant l'aspect fondamental de l'expression, à savoir l'annonce du "cocuage". De plus, le traducteur s'ingénie à effacer toute trace de cet élément, qui est pourtant au centre du récit français, en employant le terme "corneta" et non pas "cuerno" qui, en espagnol aussi bien qu'en français, désigne l'adultère, si bien que "cocu" se traduit par "cornudo", alors que l'expression lexicalisée est "cuerno de Amaltea" et non pas "corneta de Amaltea". De même, l'expression "corne d'abondance" se traduira "cuerno de la abundancia" ou "cornucopia", mais en aucun cas par le terme "corneta" qui désigne le "cornet" ou le "clairon", c'est-à-dire un instrument de musique. Ainsi, dès les premières lignes, le traducteur transforme le texte de façon significative au nom de la bienséance.

Tout au long de la nouvelle, on observe l'élimination de termes ou expressions renvoyant de façon trop explicite à la sexualité. Par exemple, l'auteur traduit la phrase "ne tâchons d'instruire la jeunesse à folier après les délices chatouilleuses de la chair" (161) par "ni tratamos de estimular a la juventud a entregarse a los extravios de una pasión que suele producir, siendo extremadas, unas consecuencias fatales" (151-152). Ici, la référence au plaisir corporel "délices chatouilleuses" est remplacée par une désignation abstraite de la relation sexuelle, doublée d'un jugement moral, "extravíos" (égarements), de même que le terme évocateur "chair" devient "pasión", terme prisé par les moralistes. Cette édulcoration s'accompagne en outre d'un ajout du traducteur qui va dans le sens de la moralisation et du jugement (" que suele producir, siendo extremadas, unas consecuencias fatales"). Le traducteur renforce donc la mise en garde de la jeunesse contre les dangers des relations charnelles, déjà présente chez Belleforest.

14. "El alcalde de Nóchera, o Nicolo, señor de Forliño" (historia trágica 4, vol. II) est la traduction de l'histoire XXIII du tome II de Belleforest: "Des grandes cruautés advenues pour l'adultère d'un des seigneurs de Nocere avec la femme du châtelain au fort de ladite cité".

15. Par la suite, toutes les citations en français sont de Belleforest.

16. Amalthée est une nymphe ayant nourri Zeus au moyen d'une corne de chèvre: c'est ainsi que serait née la corne d'abondance. 
On retrouve cette même pudibonderie du traducteur dans de nombreux exemples: ainsi, Pérez Zaragoza remplace l'expression "poursuivre sa pointe" (162), qui signifie littéralement "continuer son dessein" 17 mais dont le terme "pointe" peut être compris de façon sexuelle, et le terme plus univoque "jouir" (162), par un seul mot tout à fait neutre: "solicitar" $(153)^{18}$. De même, on observe le passage significatif de l'expression assez grivoise "sous prétexte de la chasse, il n'était semaine qu'il ne vint visiter la Garenne de la Châtelaine" (166) à une formulation beaucoup plus vague et par là même plus convenable - mais également moins spirituelle car l'on perd la métaphore de la chasse :"bajo el pretexto de la caza, no pasaba una semana sin ir a visitar a su amada Flora"-(156). La traduction, en devenant plus décente et adaptée au lectorat espagnol, perd donc en spiritualité et en richesse.

Le critère de transformation du texte dans la traduction est mis en scène par le traducteur lui-même: en effet, il traduit la phrase "il fait couper les [...] parties honteuses" (174) par "le hizo una operación cruel que el pudor me obliga a callar" (165). Là où le texte français était explicite, le traducteur, au lieu de traduire l'expression "parties honteuses" littéralement par "pudendas", utilise une périphrase explicative qui introduit un commentaire justifiant son choix d'écriture, fondé sur la bienséance. Ici, l'auteur met en avant l'élément fondamental de la pudeur qui représente une contrainte qu'il s'impose, soit par peur de la censure éditoriale soit par respect du public auquel il s'adresse. C'est bien la pudeur (et l'impudeur) qui est au centre du récit et de la traduction: ainsi, le terme fort et familier "paillardes" $(160)^{19}$, employé par Belleforest, est remplacé par Pérez Zaragoza par la périphrases "mujeres impúdicas" (150). Le terme "paillard" n'a certes pas d'équivalent direct en espagnol mais le traducteur a tout de même préféré passer par un antonyme composé du préfixe négatif "im-" plutôt que par un synonyme tel que "licencioso", "lascivo", "lúbrico", etc. Le traducteur démontre donc une réticence non seulement à évoquer ce qui tourne autour de la sexualité mais aussi à prononcer les termes qui y renvoient directement, comme si la pudeur concernait aussi bien le signifiant que le signifié.

17. "Poursuivre sa pointe": "continuer son dessein, l'entreprise qu'on a faite avec la mesme chaleur, la mesme vigueur qu'on l'a commencée" Dictionnaire de l'Académie française, 1ère Edition (1694).

18. "Le seigneur Nicole [...] délibéra de poursuivre sa pointe, et jouir de celle, les beautés et bonnes grâces de laquelle l'avaient blessé au plus profond de son âme" (162) devient "el caballero Nicolo, prendado de su hermosura y de su buena voluntad, determinó solicitarla" (153).

19. "Paillard" : "luxurieux, impudique", Dictionnaire de l'Académie française, 1ère Edition (1694). 


\section{Les consequences de la dés-érotisation sur le sens du texte: la traduction comme réécriture}

Or, cette atténuation des connotations sexuelles, loin d'être fortuite, entraîne un véritable changement de sens de l'histoire tragique. L'inflexion est déjà présente dans la transformation du titre: en effet, la nouvelle de Belleforest s'intitulait "Des grandes cruautés advenues pour l'adultère d'un des seigneurs de Nocere avec la femme du châtelain au fort de ladite cité" alors que Pérez Zaragoza intitule son récit "El alcalde de Nóchera, o Nicolo, señor de Forliño". Le titre français met ainsi en avant la structure de l'histoire, fondée sur une relation de cause à effet, l'adultère étant à l'origine des actes de cruautés; à l'inverse, le titre espagnol se contente de présenter de façon neutre deux des protagonistes de l'intrigue situés l'un par rapport à l'autre par une simple relation hiérarchique. De ce titre, on ne peut rien déduire de l'histoire qui va suivre alors que le titre français était tout à fait explicite. De plus, Pérez Zaragoza fait disparaître de son titre le personnage féminin qui se trouve pourtant au cœur de l'intrigue et dont la fonction narrative était clairement établie par Belleforest dans son titre: il s'agit d'un indice d'écart dans le traitement de ce personnage entre le texte-source et sa traduction.

Ainsi, Pérez Zaragoza non seulement estompe la dimension sexuelle de la relation adultérine mais la transforme en une histoire d'amour, là où elle n'était dans le texte français qu'une relation lascive. Du côté du protagoniste masculin, chez Belleforest, il "regarda trop lascivement la femme de son châtelain" (162) alors que chez Pérez Zaragoza, Nicolo "miró un poco atento y aficionado a Flora, mujer de su alcalde" (152). Mais cette évolution est particulièrement frappante dans la caractérisation du personnage féminin. La châtelaine de Belleforest représente le type anonyme de la femme lascive et crue en paroles:

[Elle] qui lui eut accordé sa requête par le seul signe de ses gestes et œillades, lui dit en riant d'une fort bonne grâce: [...] Vous voyez ici la Damoiselle du monde la plus prête à vous obéir, qui vive et qui pour vous complaire, sacrifiera un jour la loyauté à la furie jalouse de son mari. (165)

A l'inverse, la femme de l'alcalde de Pérez Zaragoza, qui cesse d'être anonyme et gagne du même coup en profondeur psychologique, apparaît comme une femme chaste et modeste, qui lutte entre son devoir d'épouse et son amour véritable pour le seigneur: "sabed que os amo igualmente; pero os suplico que no hagáis uso de esta tímida declaración para afligirme" (155). On peut observer un fort contraste dans leur attitude, entre le rire franc d'un côté et le sourire timide et rougissant de l'autre. L'écart est tout aussi grand dans les discours et les intentions 
qu'ils sous-tendent: dans le texte français, le discours représente une invitation ouverte à la consommation de l'adultère, mais de façon discrète: "Je veux tout ce qu'il vous plaira, moyennant que ce soit sans scandale: car j'aimerais autant mourir, comme si quelqu'un nous surprenait en nos privées et secrètes folâtries" (165). La traduction transforme ce discours en une déclaration d'amour accompagnée d'une exhortation à la vertu: "Lo principal es mi reputación, y ésta sólo podrá conservarse por la virtud" (155).

De même, le traitement de l'adultère lui-même est tout à fait différent dans le texte-source et dans sa traduction. Si le premier ne laisse aucun doute sur sa consommation, employant des termes suggestifs et s'étendant sur la description, le texte espagnol est plus concis et plus vague. La phrase "qu'un jour que les amants étaient aux prises, et au plus étroit et secret embrassement de leur lutte, il les vit accouplés d'autre lesse (sic) qu'il n'eût point souhaité, et collés plus étroitement que la raison ni honnêteté ne permettait, ni à l'un ni à l'autre" (167) devient “un día que su mujer y Nicolo estaban solos en la mayor confianza" (157).

Que l'adultère soit consommé ou non dans la traduction, le personnage féminin est blâmé de la même façon que dans l'original ${ }^{20}$, mais la conversion de la relation sexuelle en relation amoureuse a néanmoins pour effet de modifier l'interprétation de la nouvelle. Depuis la fin du XVIIIème siècle, le sentiment amoureux, souvent hors mariage, est valorisé, ce qui entraîne ici la transformation des amants coupables en victimes. En décrivant une histoire d'amour, le narrateur de la Galería fúnebre, malgré ses protestations finales, permet un mécanisme d'identification du lecteur au couple d'amoureux. Ce processus était déjà présent chez Belleforest mais plus tard dans la narration, lorsque les amants sont persécutés de façon inhumaine par le mari. Chez Pérez Zaragoza, l'identification a lieu avant même la persécution, ce qui a pour effet de perturber l'équilibre des responsabilités. En effet, bien que le narrateur de l'histoire française désapprouve la disproportion entre la faute de la femme et le châtiment infligé par le mari, ce qu'il condamne surtout chez celui-ci, c'est qu'il se venge non seulement sur l'auteur de la faute mais aussi sur sa famille pourtant innocente de tout crime. L'assassinat de l'amant est presque justifié: "plusieurs trouveraient bon le meurtre commis sur Nicole" (175) et il l'aurait été plus encore si l'alcalde avait agi sur le moment, la fu-

20. "Tal fue el fin de estos desgraciados amores que pueden servir de ejemplo a todas las mujeres para no exponerse a semejantes consecuencias por faltas a sus deberes y a su honor, convenciéndose de que no hay placer, por grande que sea, que, dando sus vueltas la rueda de la fortuna, no cause sinsabores, no estando fundado en el honor y la virtud "(180). 
reur étant un bon prétexte, plutôt que plus tard. Observons l'évolution de cette phrase chez Pérez Zaragoza: le sujet indéfini exprimant une pluralité "plusieurs" devient un sujet singulier déterminé par un adjectif indiquant un jugement de valeur dépréciatif du narrateur, "algún temerario". Par ailleurs, le conditionnel exprimant une modalisation en français est rendu en espagnol par un futur ayant la même valeur, mais auquel le narrateur ajoute une seconde modalisation au moyen de l'adverbe "quizás". Ainsi, en réduisant l'éventuel sujet d'une approbation, en le qualifiant de façon négative, en redoublant la modalisation du verbe, le narrateur de la Galería fúnebre marque clairement, et de façon beaucoup plus nette que celui des Histoires tragiques, sa distance face à ce jugement: implicitement, par opposition à cet éventuel sujet, il condamne donc le meurtre du seigneur. L'écart entre les positions des narrateurs des deux histoires est également visible dans l'appréciation de la fureur de l'instant comme motif d'excuse à la vengeance. Là où le texte français l'envisage comme unique excuse: "autrement n'y a rien qui puisse coulourer tel vice" (175), le texte espagnol dit que "no hay cosa que pueda hacer excusables estos horrorosos atentados" (166), c'est-à-dire que le traducteur est plus sévère dans son jugement du personnage. On observe donc dans cette nouvelle que certains éléments dans le récit ainsi que dans le discours, favorisent apparemment une réévaluation de la finalité de la nouvelle chez Pérez Zaragoza.

Cependant, il faut également remarquer qu'à la fin du récit, Pérez Zaragoza traduit de façon fidèle le discours commentatif du narrateur à propos de son histoire: dans cette conclusion, Belleforest rappelle les fautes et responsabilités de chacun dans le déroulement de la tragédie, des amants au mari en passant par Conrad, qui a fait preuve d'autant de cruauté que le châtelain en faisant assassiner le père de celui-ci, tout aussi innocent que le frère de l'amant tué par le châtelain. Or, le traducteur reproduit fidèlement cette admonestation collective qui n'épargne pas les amants dont la passion semblait pourtant moins fautive que chez Belleforest. Plus qu'une incohérence de Pérez Zaragoza, il faudrait y voir une tension entre plusieurs intentions de l'auteur: celle de l'écrivain de satisfaire l'identification de son public, surtout féminin, aux personnages par la description d'une passion amoureuse, bien que coupable; et celle du moraliste, cherchant l'édification à travers l'exemple (le récit), ou à défaut, celle de l'écrivain-éditeur, cherchant à contourner la censure inquisitoriale au moyen d'une déclaration de bonnes intentions.

En effet, la dés-érotisation peut être en partie expliquée par le contexte de publication de la Galería fúnebre. Le règne de Ferdinand VII était encore marqué par une forte censure gouvernementale et inquisitoriale qui veillait à la purgation ou à l'interdiction de tout écrit considéré subversif dans les domaines politique, moral 
et religieux (González Palencia 1934-1941). Cependant, la censure institutionnelle était plus modérée à la fin du règne entre la fin des années 1820 et le début des années 1830, ce qui permet de comprendre la non-interdiction de la Galería fúnebre, texte souvent subversif ${ }^{21}$ malgré les protestations de l'auteur ${ }^{22}$ et la pudibonderie que nous avons mise en avant.

Si la crainte de la censure néanmoins toujours présente peut contribuer à expliquer les choix du traducteur, on peut aussi imaginer que le phénomène de désérotisation répond à un désir de ne pas choquer un lectorat majoritairement féminin et/ou à des exigences personnelles du traducteur, qu'elles soient d'ordre moral ou esthétique. Ainsi, dans Roman et censure ou la mauvaise foi d'Eros, Maurice Couturier indique que les écrivains qui traitent de la sexualité ont toujours eu des scrupules de deux sortes, soit la peur de choquer, soit celle de tomber dans la vulgarité (Couturier 1996: 13). Dans les deux cas, il est difficile de distinguer la part de conviction personnelle de l'écrivain de la volonté de ne pas choquer le public.

Dans tous les cas de figure (volonté de détourner la censure institutionnelle, pudibonderie personnelle de l'auteur, désir de ne pas choquer son public), il s'agit d'un phénomène d'autocensure qui mime le mécanisme de censure de l'inconscient par le surmoi, dont parle Freud ${ }^{23}$. Cependant, le phénomène de censure n'est pas complet puisque Pérez Zaragoza a tout de même décidé d'inclure ce récit dans son recueil, même s'il le transforme de façon assez significative. Cela démontre une certaine part de "mauvaise foi", selon l'expression de Maurice Couturier: selon lui, les écrivains attirent inconsciemment l'attention sur les zones troubles de leur texte lorsqu'ils font preuve d'une mauvaise foi excessive. Ici, la pudibonderie appuyée de Pérez Zaragoza pourrait être comprise comme un artifice rhétorique visant à démontrer la bonne foi et la bonne morale de l'auteur alors que sa véritable intention serait subversive.

21. La dimension subversive se manifeste essentiellement dans deux domaines: la peinture de la violence ou de la cruauté, et de la sexualité, les deux étant parfois associés (voir par exemple le viol post-mortem d'une jeune vierge dans "Miladi Herwort", historia trágica 2, vol. I, qui est la traduction de "Onzièmes Ombres. Le boucher anglais, ou la lampe effrayante. Anecdote réelle", Ombres sanglantes, tome II).

22. Dans le "Prolegómeno del autor a sus lectores", Pérez Zaragoza met en avant la mission édificatrice de son ouvrage: "envuelve, bajo el velo de la historia, lecciones de la más austera moral" (48).

23. Freud introduit le mécanisme de la censure dès sa première topique (L'Interprétation des rêves, 1900) mais il ne parle de "surmoi" que dans la seconde (Au-delà du principe de plaisir, 1920; Le moi et le ça, 1923). 
Pérez Zaragoza renforce donc l'ambigüité déjà présente dans l'histoire de Belleforest, dans laquelle l'imbrication des responsabilités compliquait le processus exemplaire: 1'histoire tragique, parce qu'elle s'inspire fréquemment d'une histoire vraie et pour intéresser le lecteur, ne prétend pas avoir la transparence ni la simplicité d'une parabole. Au contraire, elle est le lieu de rencontre des conflits, aussi bien au niveau diégétique (entre les différents personnages) que méta-diégétique (conflits entre les différentes intentions, fondamentalement morales et esthétiques). Pérez Zaragoza, loin de simplifier la narration ou de rendre parfaitement cohérents récit et discours, élargit la brèche interprétative des histoires tragiques déjà mise en évidence. La dés-érotisation est donc loin d'être un élément accessoire relevant simplement d'une pudibonderie du traducteur. Au contraire, elle introduit un mécanisme mettant en jeu l'auteur et son récepteur (qu'il s'agisse des lecteurs (lectrices) ou de l'institution censoriale), qui enrichit une œuvre déjà complexe: sous couvert d'une moralisation de l'histoire tragique, qui devrait entraîner une compréhension plus directe, le texte devient en réalité le lieu de l'ambigüité et l'objet de plusieurs lectures possibles.

\section{Bibliographie}

ALONSO SEOANE, M. J. (2007). “La poderosa Themis y la Galería fúnebre: una polémica en prensa en el contexto de la traducción de colecciones de relatos en España (1830-1831) ", Anales de Filología Francesa 15: 5-16.

ALONSO SEOANE, M. J. (2010). “ Nuevos datos sobre la Galería fúnebre de Agustín Pérez Zaragoza y algunos aspectos de la repercusión de su obra en la prensa " in Actas del XVI Congreso de la Asociación Internacional de Hispanistas: Nuevos caminos del hispanismo, París, del 9 al 13 de julio de 2007. (Coords. Pierre Civil et Françoise Crémoux). Madrid: Iberoamericana; Frankfurt: Vervuert.

ANONYME (1603). Historias trágicas exemplares, sacadas del Bandello veronés. Nuevamente traducidas de las que en lengua Francesa adornaron Pierre Bouistau (sic) y Francisco de Belleforest. Contiénense en este libro catorce Historias notables, repartidas por capitulos. Valladolid: Lorenzo de Ayala.

ARNOULD, J.-C. (2003). "L'impasse morale des histoires tragiques au XVIème siècle", Bulletin de l'Association d'étude sur l'humanisme, la réforme et la renaissance 57: 93-108.

BANDELLO, M. (1554-1673). Novelle. (4 vols.).

BELLEFOREST, F. de (1566). Le Second Tome des Histoires tragiques, extraites de l'italien de Bandel, contenant encore dix-huit Histoires traduites et enri- 
chies outre l'invention de l'Auteur, dédié à Ma Damoiselle la Procureuse générale. Paris: Robert le Magnier.

CASTEllanOS DE LOSADA, B. S. et ANENTO, J. (1830). La Poderosa Themis, o los remordimientos de los malvados. Obra escrita por Monsieur David. (2 vols.). Madrid: Imprenta de D. Ramón Verges.

COUTURIER, M. (1996). Roman et censure ou la mauvaise foi d'Eros. Seyssel: Champ Vallon.

CUENCA, L. A. de (1977). "Prólogo" a Galería fúnebre de espectros y sombras ensangrentadas. Madrid: Editora Nacional: 15-40.

CUISIN, P. (1820). Les ombres sanglantes, Galerie funèbre de Prodiges, Evènements merveilleux, Apparitions nocturnes, Songes épouvantables, Délits mystérieux, Phénomènes terribles, Forfaits historiques, Cadavres mobiles, Têtes ensanglantées et animées, Vengeances atroces et combinaisons du crime, puisés dans des sources réelles. Recueil propre à causer les fortes émotions de la terreur. (2 vols.). Paris: Veuve Lepetit.

GONZALEZ PALENCIA, Á. (1934-1941). Estudio histórico de la censura gubernativa en España (1800-1833). Madrid: Tipografía de Archivos.

LARRA, M. J. (1995). Artículos. Madrid: Olympia.

LÓPEZ SANTOS, M. (2010). La novela gótica en España (1788-1833). Vigo: Academia del Hispanismo.

PECH, T. (2000). Conter le crime. Droit et littérature sous la Contre-Réforme: les histoires tragiques (1559-1644). Paris: Champion.

PÉREZ ZARAGOZA GODÍNEZ, A. (1831). Galería fúnebre de historias trágicas, espectros y sombras ensangrentadas. Obra nueva de prodigios, acontecimientos maravillosos, apariciones nocturnas, sueños espantosos, delitos misteriosos, fenómenos terribles, crímenes históricos y fabulosos, cadáveres ambulantes, cabezas ensangrentadas, venganzas atroces, y casos sorprendentes. Colección curiosa e instructiva de sucesos trágicos para producir las fuertes emociones del terror, inspirando horror al crimen, que es el freno poderoso de las pasiones. (12 vols.). Madrid: Imprenta Palacios.

PÉREZ ZARAGOZA GODÍNEZ, A. (1977). Galería fúnebre de espectros y sombras ensangrentada. (Ed. de Luis Alberto Cuenca). Madrid: Editora Nacional.

ROAS, D. (2001). La recepción de la literatura fantástica en la España del siglo $X I X$. Tesis doctoral. Universitat Autònoma de Barcelona.

ZERARI PENIN, M. (2012). "Le "cerveau noir" des nouvellistes. Remarques chromatiques sur la nouvelle post-cervantine", in Les couleurs dans l'Espagne du Siècle d'or. Ecriture et symbolique. (Dir. Yves Germain et Araceli Guillaume-Alonso). Paris: PUPS: 267-285. 